\title{
Pre-operative imaging and post-operative appearance of standard paediatric neurosurgical approaches: a training guide for neuroradiologists
}

\author{
Mario Ganau^, Shailendra A. Magdum, Amedeo Calisto \\ Department of Neurosurgery, Oxford University Hospitals NHS Foundation Trust, Oxford, UK \\ Contributions: (I) Conception and design: M Ganau; (II) Administrative support: All authors; (III) Provision of study materials or patients: A Calisto; \\ (IV) Collection and assembly of data: M Ganau; (V) Data analysis and interpretation: SA Magdum; (VI) Manuscript writing: All authors; (VII) Final \\ approval of manuscript: All authors. \\ Correspondence to: Mario Ganau, MD, PhD, MBA, FEBNS, FACS. Department of Neurosurgery, Oxford University Hospitals NHS Foundation \\ Trust, Headley Way, OX3 9DU, Oxford, UK. Email: Mario.Ganau@alumni.harvard.edu.
}

\begin{abstract}
A short-cut narrative review was conducted according to the SANRA guidelines to identify studies describing normal and abnormal postoperative radiological features of the most common paediatric neurosurgical procedures. Rather than focusing on the original pathology addressed by neurosurgical means, this review explored three main areas of operative neurosurgery: ventricular access, supratentorial \& infratentorial craniotomies, and posterior fossa/craniocervical junction decompression. A total of twenty-three landmark papers were included for review based on their relevance to address the research question and serve as a practical guide for paediatric neuroradiology trainees and fellows. Accurate in text referencing of the ClinicalTrials.gov identifier, and weblink, has also been provided for all trials discussed in the results section. All the above is complemented by relevant iconography meant to describe a wide range of postoperative changes and early complications. Finally, the review is enriched by a discussion touching upon haemostatic agents, intentionally retained foreign bodies and the future of machine learning for neuroradiology reporting. Overall, the information presented in a systematic fashion will not only help trainees and fellows to deepen these topics and expand their knowledge in preparation for written and oral boards, but will also represent a useful resource for everyone including trained neuroradiologists and neurosurgeons themselves.
\end{abstract}

Keywords: Neuroimaging; postoperative complications; neuroradiology reporting; medico-legal; artificial intelligence

Submitted Dec 30, 2020. Accepted for publication Mar 03, 2021.

doi: 10.21037/tp-20-484

View this article at: http://dx.doi.org/10.21037/tp-20-484

\section{Introduction}

The responsibility of paediatric neuroradiologists primarily includes the indication for various imaging investigations, the quality of the diagnosis, the range of differentials provided, and the communication of the results to the requesting clinician (1). Those basic tasks have wider implications for the multidisciplinary team, as they will often guide the information released to patients and relatives, change treatment courses (tackling of postoperative complications, residual tumours, etc.), and might also lead to medico-legal entanglement. Keeping that

\footnotetext{
^ ORCID: 0000-0002-8676-1147.
} 
in mind, this article was conceived to provide paediatric neuroradiologists and neurosurgeons with a practical guide covering the indications, risks and benefits of the most common neurosurgical procedures, and support in preparing and interpreting the reports of postoperative images. The idea behind such research project was to provide exemplificative cases in parallel to a shortcut narrative review of the literature, which has been conducted to identify studies investigating normal and abnormal postoperative radiological aspects. Rather than focusing on the original pathologies (normally classified as neuro-oncology, neuro-vascular, cerebrospinal fluid dynamics, infections, etc.) addressed by neurosurgical means, this study will explore three main areas of operative neurosurgery, whose indication might overlap for various pathological conditions: ventricular access, supratentorial \& infratentorial craniotomies, and posterior fossa/ craniocervical junction decompression.

\section{Methodology}

A narrative review of the relevant literature was conducted on the following electronic databases: PubMed, Ovid Medline, Scopus, Cochrane Library, US National Library of Medicine-ClinicalTrials.gov; National Institute for Health and Care Excellence - NICE, Google Scholar and Embase. The following $\mathrm{MeSH}$ terms, including combination of them, were used: "ventricular access device", "intracranial ventricular trajectory", "third ventriculostomy", "ventricular drainage", "supratentorial craniotomy", "infratentorial craniotomy", "pterional craniotomy", "interhemispheric approach", "suboccipital craniectomy", "posterior fossa decompression", "postoperative complication".

No language restrictions were applied, and the search was extended from 1990 up to 2020. Clinical trials, systematic reviews, meta-analysis, and case series were retained for consideration of inclusion; case reports, editorials and letters to the editors were automatically excluded from further assessment. The initial search was conducted with the assistance of a research librarian, and only landmark papers, or those with a specific paediatric neuroradiology perspective, were initially triaged. The first author was responsible for initial selection of articles to be included in this review; whenever doubts arose regarding the relevance of selected papers the final decision regarding inclusion was taken through a Delphi approach among all authors. Exemplificative cases were selected from our own surgical practice to enrich the educational content of this article.

To ensure quality assessment of this review, its design and reporting have been conceived and conducted in agreement with the SANRA guidelines for narrative review articles (2). Accurate in the text referencing of the ClinicalTrials.gov identifier and weblink has been provided for all trials discussed in the results section.

\section{Results}

Through the review of the literature a total of twentythree studies were found suitable to meet the purposes of this narrative review. Among the identified articles, six were pertaining to ventricular access, ten revolved around supratentorial \& infratentorial craniotomies, while seven described posterior fossa/craniocervical junction decompression.

\section{Ventricular access}

Various techniques have been described for the insertion of temporary or permanent ventricular access catheters (VAC). Nowadays the use of intraoperative neuronavigation is considered the gold standard for insertion of VAC in adults (3), and paediatric neurosurgery is no exception, as stated by the Paediatric Hydrocephalus Systematic Review and Evidence-Based Guidelines Task Force (4). However, when neuronavigation is unavailable the neurosurgeon must rely upon craniometrics parameters to achieve successful ventricular cannulation. Morone et al. wrote a historical review, summarising the most standardised ventricular access points, namely the Kocher's, Kaufman's, Paine's, Menovksy's, Tubbs', Keen's, Frazier's, Dandy's, and Sanchez's points (5). Of note, craniometric calculations have been the very essence of neurosurgery over the past centuries, and a thorough understanding of the correlation between sutures, suture junctions, prominences/depressions of the calvaria, and cortical surface landmarks is essential also for diagnostic radiologists (6). Consequently, although the description of each different entry point goes beyond the scope of the present review, our readers can refer to the abovementioned historical review for a comprehensive description of the relevant craniometric calculation and related intracranial trajectories to the ventricular system. Not only those entry points are routinely used for keyhole insertion of either temporary or permanent ventricular devices for cerebrospinal fluid (CSF) sampling and diversion, such as drains (Figure 1) and shunts (Figure 2), but also for 

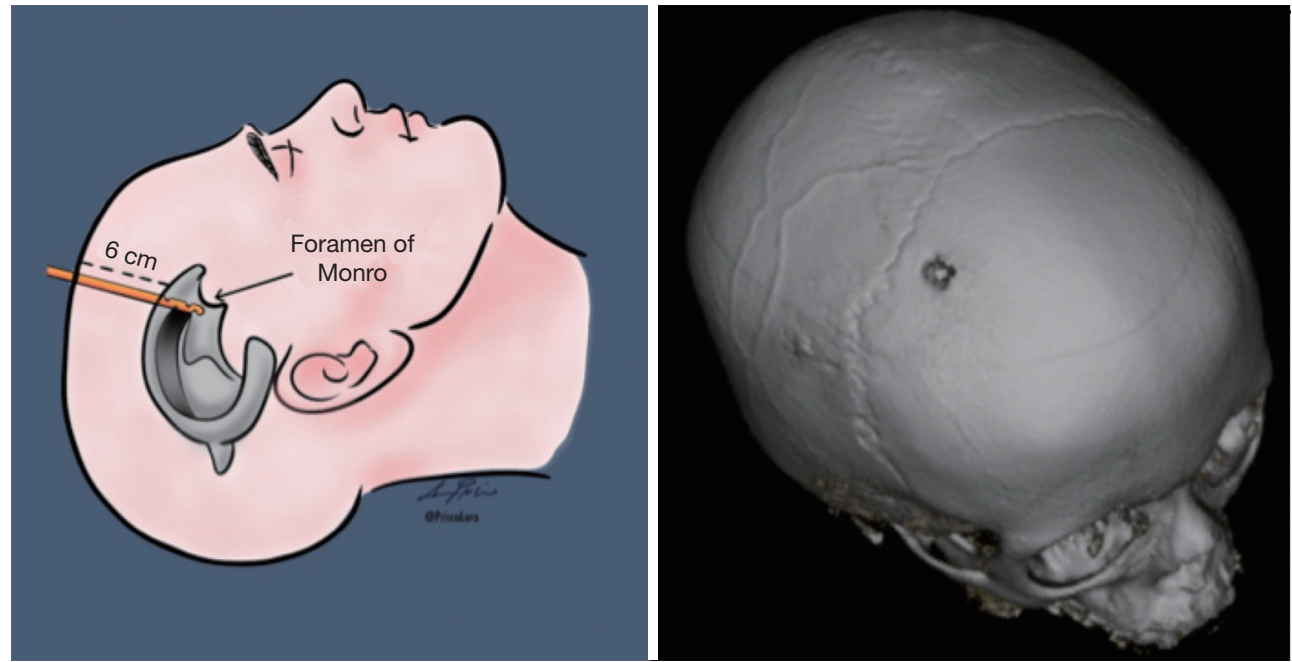

Figure 1 Craniometrics coordinates for Kocher's point (Left image) and overview of burr hole on postoperative 3D CT scan (Right image).
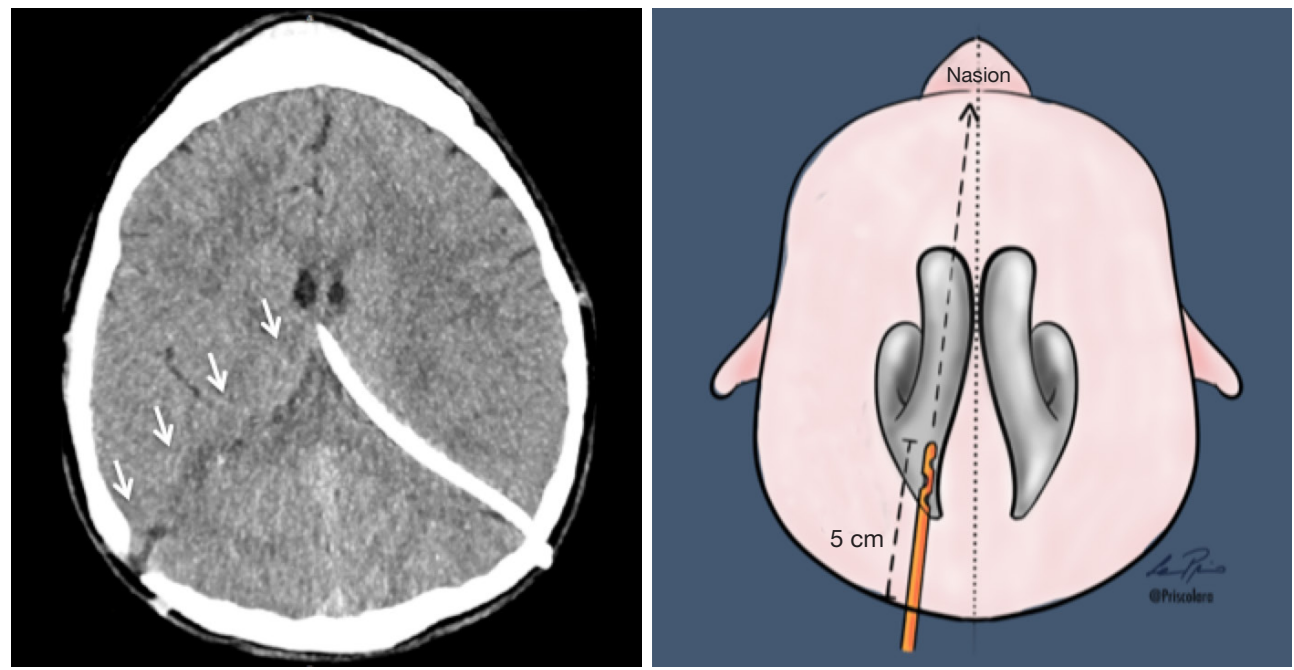

Figure 2 Revision of ventriculoperitoneal shunt using Keen's point (Left image, axial CT scan, please note the white arrows indicating the previous trajectory) compared to craniometrics coordinates for ventricular access through Frazier's point (Right image, please note the black arrow indicating the direction aimed during ventricular cannulation).

evacuation of intraventricular clots, coagulation of choroid plexus, biopsies of ventricular lesions, and intrathecal administration of drugs or other chemotherapeutics. Due to the role played by various neuro-oncology and neurovascular pathologies in altering the CSF dynamics, VAC can be required in a number of clinical scenarios; this said, in paediatric neurosurgery the most common indication is represented by congenital hydrocephalus whose incidence is high (1/500 births). Other forms of acquired hydrocephalus (i.e., secondary to infections, post-traumatic intracranial/intraventricular bleeding, etc.), although less common, can also be encountered (7). As such, paediatric neuroradiologists may be asked to provide insights into some of the primary reasons why hydrocephalus occurs or persists despite surgical treatment (8). To this regard, an index of suspicion should therefore be kept for the following scenarios: blockage of the CSF flow, excessive production or impaired resorption, slit ventricles, association with other malformations (i.e., agenesia of midline structures, abnormalities of the posterior fossa, neural tube defects, 

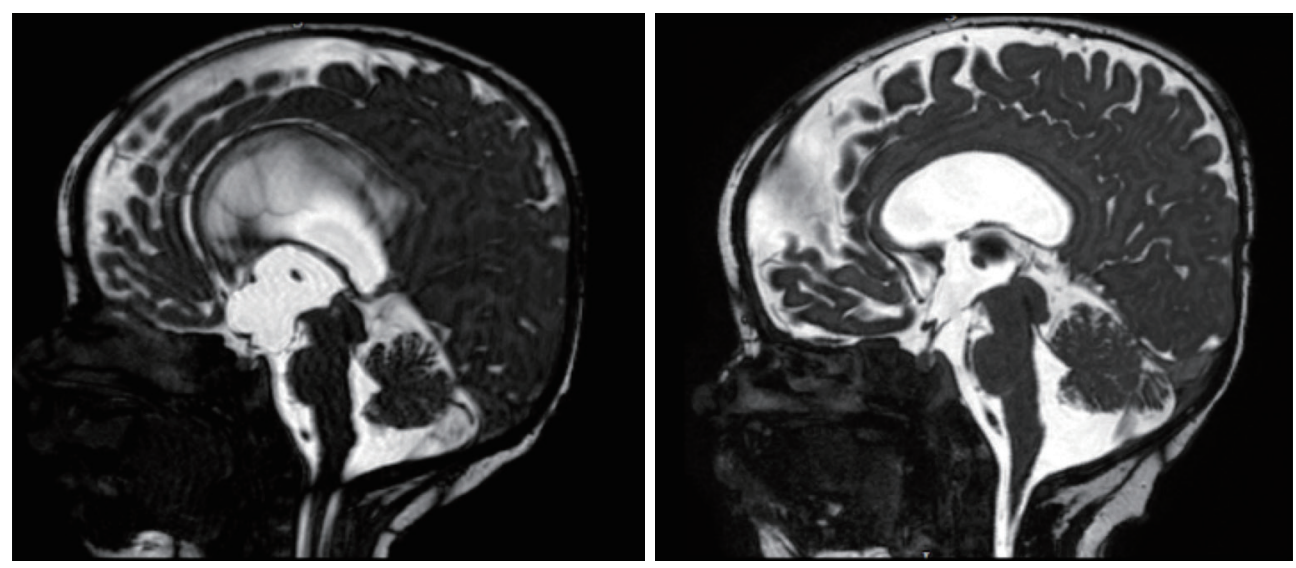

Figure 3 Sagittal FIESTA MRI scan of 3 years old patient undergoing endoscopic third ventriculostomy (ETV) for acqueductal stenosis (Left image, preoperative scan showing the radiological signs of raised intracranial pressure) with resolution of clinical signs of raised intracranial pressure (Right image, postoperative scan showing flow void and turbulent cerebrospinal fluid flow through the artificial communication created in the floor of third ventricle, with a concomitant relaxed appearance of the third ventricle).

etc.). Beside an urgent $\mathrm{CT}$ head to rule out acute hydrocephalus (or trans-fontanellar ultrasound in neonates), a battery of investigations might be warranted: anteroposterior (AP) and lateral (LL) $\mathrm{x}$-rays of the entire shunt in adjunct to the computed tomography (CT) scout imaging (a.k.a. shunt series to assess tubing disconnection or change in the valve setting), ultrasound of the distal end (abdomen, bladder, lung/pleural interface, subgaleal region, ECO cardio to assess the presence and nature of collections), and cine magnetic resonance imaging (cineMRI) with Constructive Interference in Steady State (CISS) and Fast Imaging Employing Steady-state Acquisition (FIESTA) sequences (to study the CSF dynamics). The results will then need to be put into context with a broad range of clinical features (particularly in neonates), such as bradycardia, anisocoria, mydriasis, nausea, vomiting, upgaze palsy, papilloedema, convergence retraction nystagmus, pupillary hyporeflexia, fluctuation in level of consciousness, increase in head circumference. What is more important to stress here is the need to provide as many clarifications as possible in the neuroradiology request regarding the patient's clinical conditions: to know whether the patient is 'well' or 'unwell' during a scan, allows to compare ventricular dimensions over time in case of future suspected episodes of blocked shunts.

Management of hydrocephalus is oftentimes performed in the form of emergency procedures that, despite considered routine in paediatric neurosurgery, are burdened by number of possible early and late complications, much of them specific to the original pathology addressed. For instance, Zairi et al. reported a $10 \%$ rate of early complications following management of hydrocephalus associated to leptomeningeal metastases from solid tumours with the use of an Ommaya reservoir connected to a VAC, those included: catheter malpositioning, intracranial haemorrhage, and superficial or deep-sited surgical site infections (SSI). Of note, in their series $72 \%$ of the recorded complications required revision surgery, and the mortality rate was $9 \%$. These figures suggest that even a very quick and uneventful surgical intervention (mean operative time for Zairi et al. was 15 minutes and the authors reported no intra-/perioperative complications) can bear devastating consequences (9). Hence the importance of a prompt radiological assessment of routine postoperative scans, recognition of possible complications, and rapid escalation to the requesting clinician as the only means to timely deal with them.

Additionally, it is worth mentioning that permanent VAC can serve various others CSF diversion purposes (as in any shunt for hydrocephalus), but can also serve as a safety-net device if connected to a reservoir after endoscopic third ventriculostomy (ETV), another surgical intervention meant to restore CSF dynamics through the ventricular and cisterns systems (Figure 3). Clinically, ETV success is defined by a lack of subsequent need for CSF diversion in form of permanent shunt procedures (10); whereas radiologically, its success is defined by the decrease in the size of lateral and third ventricles, and the presence of flow void signal 


\begin{tabular}{|c|c|c|c|}
\hline & Anterior fossa & Middle fossa & Posterior fossa \\
\hline & \multicolumn{2}{|c|}{$\begin{array}{l}\text { Pterional craniotomy } \\
\text { (used to access lesions affecting the Sylvian fissure, frontal and temporal lobes, circle of } \\
\text { Willis, cavernous sinus, superior orbital fissure, sellar and suprasellar lesions) }\end{array}$} & \multirow{2}{*}{$\begin{array}{l}\text { Suboccipital craniotomy } \\
\text { (used to access the cerebellar hemispheres, roof and floor } \\
\text { of the fourth ventricle, antero-lateral brainstem, posterior } \\
\text { aspect of the petrous bone, and upper cervical spinal cord) }\end{array}$} \\
\hline & \multicolumn{2}{|c|}{$\begin{array}{l}\text { Convexity and parasagittal approaches } \\
\text { (used for superficial lesions along the convexity or the calvarial vault, while preserving } \\
\text { parenchymal, venous and arterial structures) }\end{array}$} & \\
\hline 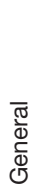 & $\begin{array}{l}\text { Frontal and bifrontal craniotomy } \\
\text { (mostly used for large pituitary tumours with } \\
\text { suprasellar extension, but also common for } \\
\text { frontal lobe resections, and management of } \\
\text { medically refractory intracranial hypertension } \\
\text { due to traumatic brain injury) }\end{array}$ & $\begin{array}{l}\text { Subtemporal craniotomy } \\
\text { (used to approach lesions located in the } \\
\text { suprasellar, parasellar \& interpeduncular } \\
\text { cisterns, including lateral aspects of the } \\
\text { midbrain and the pons). }\end{array}$ & $\begin{array}{l}\text { Retrosigmoid Craniotomy } \\
\text { (provides the simplest access to the cerebellopontine angle } \\
\text { and its content, including the V, VII, VIII, IX, X and XI cranial } \\
\text { nerves. It can be extended upwards and downwards to } \\
\text { supratentorial and craniocervical regions) }\end{array}$ \\
\hline & $\begin{array}{l}\text { Interhemispheric approach } \\
\text { (used to access lesions of the corpus } \\
\text { callosum or the forth ventricle) }\end{array}$ & \multicolumn{2}{|c|}{$\begin{array}{l}\text { Combined anterior and posterior petrosectomy } \\
\text { (used to access the entire petroclival region, anterior brainstem and the internal auditory canal while } \\
\text { avoiding incision of the tentorium, and decreasing the need for temporal lobe or cerebellar retraction) }\end{array}$} \\
\hline 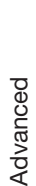 & $\begin{array}{l}\text { Orbitozygomatic craniotomy } \\
\text { (used to access anterior circulation and } \\
\text { pathology of the sellar, cavernous sinus, } \\
\text { mesial temporal lobe and subfrontal region) }\end{array}$ & $\begin{array}{l}\text { Translabyrinthine approach } \\
\text { (rarely used for internal auditory canal } \\
\text { and cerebellopontine angle lesions, } \\
\text { oftentimes considered for revision surgery } \\
\text { in care of remnants from previous middle } \\
\text { fossa approaches) }\end{array}$ & $\begin{array}{l}\text { Presigmoid craniotomy } \\
\text { (provides the shortest distance to the petroclival region, and } \\
\text { is usually considered to access the vertebrobasilar junction, } \\
\text { anterior brainstem and cranial nerves in the anterior portion } \\
\text { of the posterior fossa) }\end{array}$ \\
\hline
\end{tabular}

Figure 4 Craniotomies for surgical approaches to Anterior, Middle and Posterior Fossae

through the floor of third ventricle on postoperative MRI. A nice article from Aquilina et al. demonstrated that routine connection of a reservoir to the VAC allows lifesaving emergency such as the therapeutic aspiration of CSF in case of sudden neurological deterioration caused by ventriculostomy failure. Furthermore, in reporting follow up scans the neuroradiologist should also be aware that permanent reservoirs can potentially assist in the diagnosis of ETV failure, CSF dynamics studies, and can in case of radiological doubts can also serve other purposes, such as intrathecal administration of contrast media (11).

\section{Supratentorial \& infratentorial craniotomies}

Although neuronavigation-assisted, minimally invasive, endoscopic surgery is on the rise, craniotomies are routinely performed to access a vast number of different intracranial space-occupying lesions, including: tumours, haemorrhages, arachnoid cysts, epileptogenic lesions, vascular malformations, abscesses, etc. (12-15) Despite the very wide range of different craniotomies that neurosurgeons can consider for their patients, the ones most commonly performed in children, and those primarily considered in paediatric neurosurgical training models, are: the pterional, interhemispheric and suboccipital craniotomies. A comprehensive description of all other craniotomies would be complex and time-consuming, hence we would direct the readers to Figure 4 for a broader synoptic view of their classification and indications, whereas in this section we will only cover the most common ones.

The greatest advantage of the pterional craniotomy consists in its versatility and familiarity of all neurosurgeons with this approach. Also known as fronto-temporal craniotomy, it allows access to most vascular lesions and tumours located in close proximity to the sylvian fissure and circle of Willis. Besides very generic risks associated with any other type of craniotomy, such as SSI, CSF fistulas, and pneumocephalus, attention must be paid to avoid excessive retraction of the frontal and temporal lobes, which could result in cerebral contusions/infarct and neurologic deficits (Figure 5). In those circumstances merging information from postoperative plain CT, CT Angiography, and MRI with Fluid Attenuated Inversion Recovery (FLAIR), Apparent Diffusion Coefficient (ADC) and Diffusion-weighted Imaging (DWI) sequences might help in the assessment to ischaemic changes and prediction of neurological recovery. Additionally the use of contrast-enhanced images should always be considered within 48-72 hours from the surgical intervention to minimise postoperative artifacts while distinguishing between degradation of blood products and tumour residual (16).

The interhemispheric transcallosal transchoroidal 

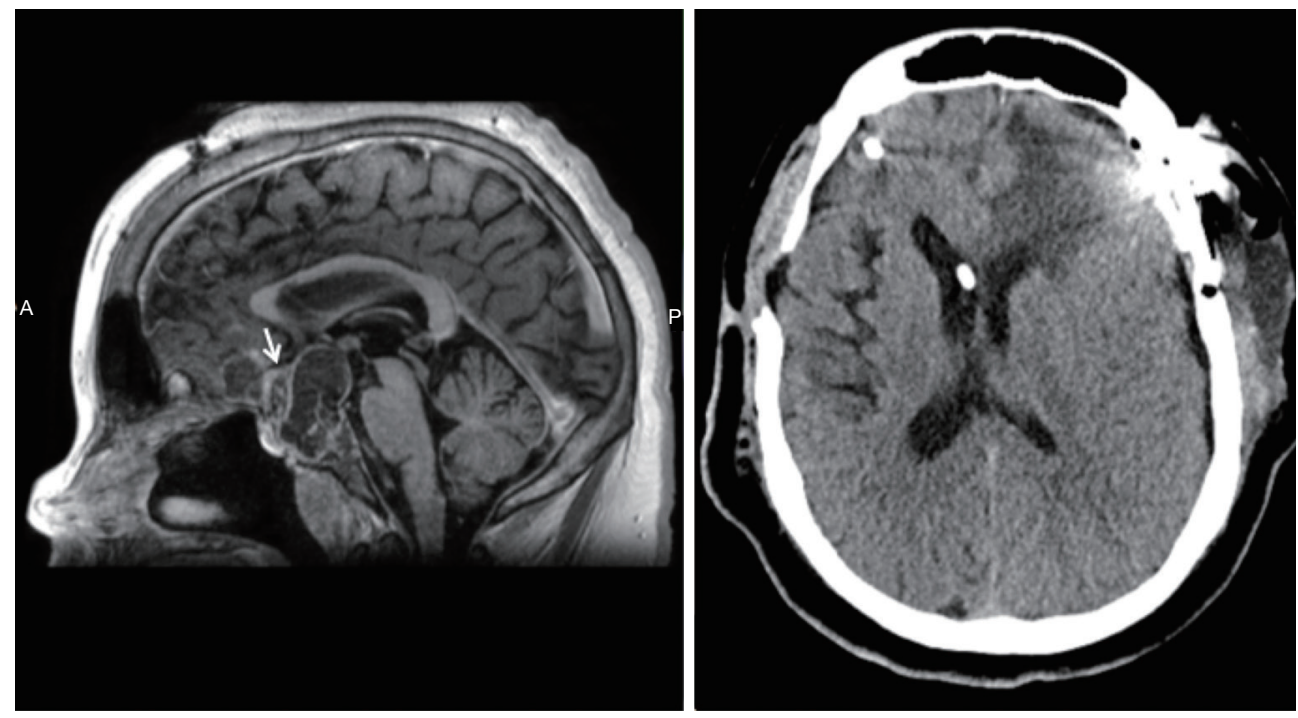

Figure 5 Preoperative and postoperative neuroradiology workup in 5 years old patient undergoing Left fronto-temporal craniotomy for craniopharyngioma following previous contralateral approach (Left image, preoperative sagittal contrast-enhanced T1WI MRI showing the suprasellar extension of the lesion; Right image, axial CT scan demonstrating intraventricular cannula in the antrum of Right lateral ventricle for shunting purposes, Right frontal parenchymal trajectory of intralesional cannula for Ommaya reservoir, Left frontal ischaemic changes following surgical retraction to access and debulk the lesion).

approach provides the shortest route to pathologies of the third ventricle, periventricular region and pineal gland, while avoiding risks associated with alternative surgical routes, such as violation of the cortical structures, parenchymal haemorrhage, and seizures (17). Whereas transcortical approaches would require large ventricles, this is not a prerequisite for interhemispheric approach, which can be considered even in case of normal ventricles (but not slit ventricles). This approach is particularly useful for centrally located lesions arising from the anterior and lateral walls of the third ventricle, even of large diameter (Figure 6). The most severe complications associated with this type of approach are related to the high risk of injury to the superior sagittal sinus, anterior cerebral arteries, corpus callosum, fornix, thalami and thalamostriate veins. As such they include intraventricular haemorrhage, delayed hydrocephalus, postoperative pneumoventriculus, meningitis, ventriculitis, venous infarction, seizures, memory loss, diencephalic syndrome, disconnection syndrome, tremor and Parinaud syndrome. Although most patients will not be left with persistent morbidity, the risk of approach-related complications is very high even in very expert hands; in fact almost 1 in 3 patients are expected to experience some complications $(18,19)$, and mortality is estimated to be $<1 \%$ (20).
Suboccipital craniotomies are commonly performed for the excision of cerebellar tumours and fourth ventricular tumours (such as medulloblastomas, ependymomas, and cerebellar astrocytomas), which are the most common location of primary intracranial tumours in paediatric patients (21). This surgical approach is burdened by many intra- and postoperative complications (22), which are very much similar to those listed for the craniotomies presented above. One very specific complication, though, is cerebellar mutism syndrome, a postoperative syndrome typically arising 1 to 2 days after resection of a midline posterior fossa tumours, which consists of diminished speech progressing to mutism, emotional lability, hypotonia, and ataxia (23). While some of the symptoms recover after a few months, neuropsychological testing shows long-term deficits in language (agrammatism), executive function and verbal memory (24). Paediatric neuroradiologists might be asked to further investigate disconnection syndromes, and in those circumstances attention should be paid to compare DTI and tractography in the pre- and postoperative scans (25). While the use of those MRI sequences is highly recommended to reveal neural connectivity changes, they still do not represent standard of care in paediatric neurosurgery. To this regards, it is encouraging to notice that a single centre case control study, NCT02810626, from University 

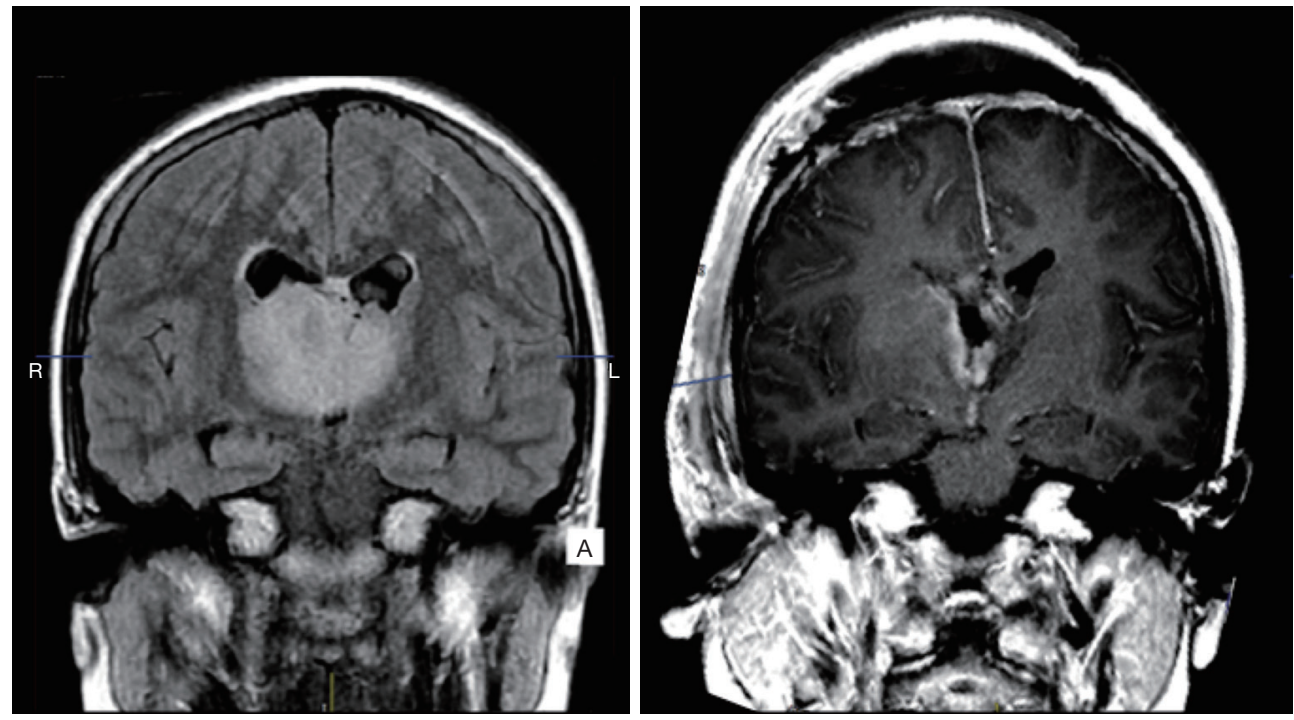

Figure 6 Preoperative and postoperative neuroradiology workup in 12 years old patient undergoing interhemispheric approach and callosotomy for debulking of Left thalamic glioma (Left image, Coronal FLAIR showing the hyperintense lesion originating from Left thalamus and invading the ventricular space with obstruction of foramens of Monro; Right image, Coronal contrast-enhanced T1WI showing some blood-related ependymal enhancement in the surgical cavity following complete resection of the lesion).

of Western Ontario (https://clinicaltrials.gov/ct2/show/ NCT02810626) is currently looking at implementing neuroradiology protocols to avoiding eloquent fiber tracts in the brain and cerebellum, thus minimizing damage pertaining to neurologic and motor functionality, in children undergoing such kind of interventions

Posterior fossa decompression: Several malformations of the craniocervical junction can be found in the paediatric population and require surgical decompression with or without stabilisation. The craniocervical junction is a biomechanically complex articulation comprising the occipital condyles, the atlas (C1) and axis (C2). A myriad of conditions can cause either biomechanical instability, and/or deformity, and/or neuraxial compression; usually they are classified as follows: congenital malformations (such as platybasia, atlas assimilation, basilar invagination, atlantoaxial fusion, os odontoideum, etc.), developmental (typically in cases of achondroplasia and Down syndrome), infections (Griesel syndrome, tubercolosis, etc.), inborn errors of metabolism (mucopolysaccaridosis, including Hunter syndrome, Morquio syndrome, Shy syndrome, etc.), traumas and tumours (26-29).

Whereas, as mentioned above, the incidence of tumours in posterior fossa is high in children, that of tumours of the craniocervical junction is relatively low. Despite such a low incidence various types of tumours have been described in this region, including chordomas of the clivus and foramen magnum, fibrous dysplasia, aneurysmal bone cysts, eosinophilic granuloma affecting the atlas and axis, Ewing's sarcoma involving the atlas, osteoblastoma, neurenteric cysts, meningioma, schwannoma and plexiform neurofibromas (30). A reporting paediatric radiologist might be called to provide analytical descriptions of those abnormalities on initial CT and MRI scans, suggest additional sequences (including dynamic CT/MRI images), and correlation with clinical findings; nonetheless an even greater challenge may be encountered when dealing with mild cerebellar tonsils ectopia which could still be physiological finding in paediatric patients up till their teens. This latter scenario represents the most common reason for referral to additional clinical, neuroradiological and neurophysiological investigations meant to rule out Chiari 1 malformation (CM-1) also known as Chiari syndrome (31). CM-1 is certainly the most commonly encountered (1/1,000 births according to the US National Institute for Neurological Disorders and Stroke, https://www.ninds.nih.gov/Topic-Areas/Chiarimalformation). A complete knowledge of the image findings not only in the posterior fossa but also in the rest of the brain and spine is critical for the optimal assessment and management of these patients (32). The pathogenesis of CM-1 is an under-development of the posterior fossa, which is narrower and cannot accommodate adequate 

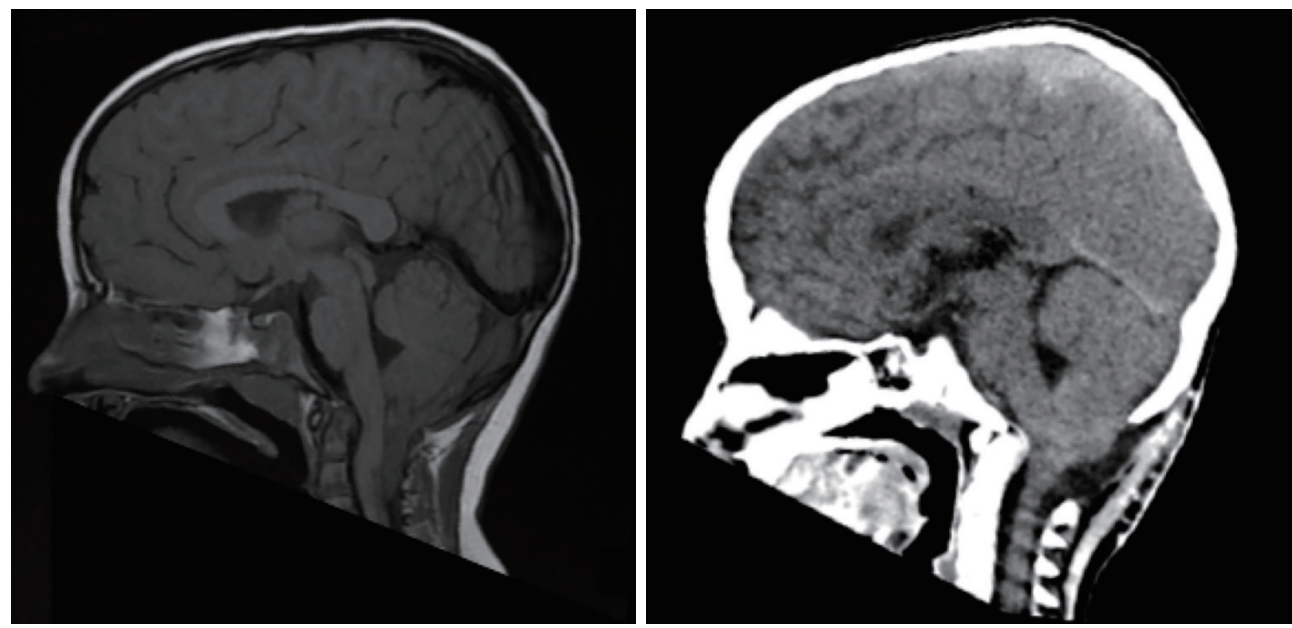

Figure 7 Preoperative and postoperative neuroradiology workup in 9 years old patient with Chiari 1 malformation (CM-1) (Left image, sagittal T1WI MRI showing a typical overcrowded posterior fossa with cerebellar ectopia through the foramen magnum down to the level of C3 and evidence of syringomyelia in the cervical spinal cord; Right image, sagittal CT scan showing mild proptosis of the cerebellum through the posterior fossa decompression without evidence of postoperative pseudomeningocele).

space for its content, therefore, similarly to patients with craniosynostoses, CM-1 patients may present with clinical features of raised intracranial pressure and often require invasive monitoring with placement of intraparenchymal probes (32). Surgeries for symptomatic CM-1 patients include posterior fossa decompression involving craniectomy with or without dural opening, and duroplasty (Figure 7), the most important aspect of postoperative imaging is: (I) to identify ongoing crowding or whether it is likely that further decompression is necessary; and (II) to document possible complications including hydrocephalus, pseudomeningocele, SSI, hygroma, etc. $(33,34)$. A recent systematic review and meta-analysis revealed that in the absence of hydrocephalus and craniocervical region instability, decompression and duroplasty provide better clinical outcomes but with higher risk, suggesting that the use of simple decompression with or without dural opening may be justified in children with higher surgical/anaesthesiological risk due to other comorbidities, and in the absence of a syrinx (35). Impact of decompression on syrinx has been carefully reviewed only in retrospective cohort studies. Chotai et al. reported that within 3 months from surgery the mean syrinx diameter regressed from $8.1 \pm 3.4 \mathrm{~mm}$ preoperatively to $5.6 \pm 2.9 \mathrm{~mm}$; whereas $\geq 50 \%$ regression in diameter was observed on average after 8 months (95\% CI: 4.2-11.8 months) (36). This study demonstrated that the syringes continue to regress over time, even though tonsil coagulation was associated with early syrinx regression in that cohort. This said, the role of surgical manoeuvres such as tonsil coagulation and arachnoid veil identification and sectioning in the overall role of posterior fossa decompression for CM-1 remains unclear, hence the rationale for the randomised clinical trial NCT02669836 sponsored by Washington University School of Medicine (https://clinicaltrials.gov/ct2/show/NCT02669836) started in 2016 and still ongoing.

\section{Discussion}

The medical liability of diagnostic neuroradiologists is obviously not the same of interventional specialists; additionally it can sometimes be shared with other clinicians, particularly neurosurgeons. Knowledge of the most common neurosurgical approaches might be a game changer facilitating reporting of postoperative investigations. An immediate recognition of normal and abnormal patterns, a deep understanding of potential clinical pitfalls and need for additional investigations allows a smooth neuroradiology workflow and timely escalation to the appropriate specialist, whenever prompt surgical or medical treatments are needed.

The advances achieved over the years in neurosurgical techniques and neuroanaesthesia have driven a parallel expansion in the array of conditions, which are potentially treatable with surgery $(37,38)$. As such, the range of surgical options is now wider than ever, and includes minimally invasive procedures amenable for service improvement 

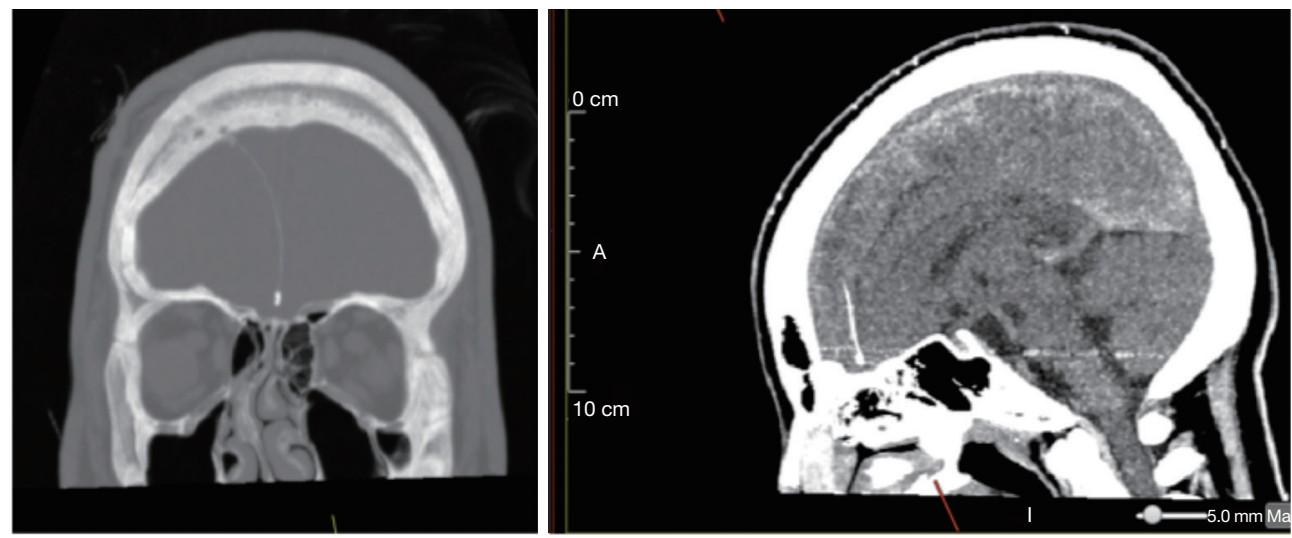

Figure 8 Postoperative CT appearance of misplaced intraparenchymal probe for intracranial pressure monitoring (Right image, coronal bone window CT scan showing the probe close to the lamina cribrosa; Left image, sagittal plain CT scan showing the trajectory of the probe toward the floor of anterior fossa).

projects focused around the concepts of early recovery after surgery and prompt discharge from neurosurgical centres (39-41). An awareness of the surgical thinking and planning of neurosurgical interventions in children, and the practical knowledge of the operative steps involved, can truly lead to enhance accuracy in radiology reporting of the postoperative scan (37).

Beside the specifics of the exemplificative cases presented, some other general aspects have to be mentioned, including the postoperative appearance of intraoperatively-used haemostatic agents, or that of intentionally retained foreign bodies. Finally an overview of the role of machine learning in neuroradiology reporting concludes the discussion section.

\section{Haemostatic agents}

Haemostatic substances are routinely used during intracranial surgery, the range of commercially available substances is very wide and comes under various forms, including sponge and powder, gelatin granules matrix, fibrillar oxidized cellulose polymer, etc. (42-44). While their use in adult neurosurgery is widely accepted, currently only one prospective, open label, multicentre randomised study, NCT02309645, is investigating the efficacy of a fibrin sealant for its use in paediatric craniotomies (https:// clinicaltrials.gov/ct2/show/NCT02309645). Although advanced haemostatics are completely absorbed within four to six weeks from surgical intervention their residual in the surgical site can mislead the reporting radiologist by affecting early postoperative imaging, causing artefacts, and simulating space-occupying masses, while in the long term can potentially lead to an overestimation of scar tissue. The mass effect caused by clot formation and granulomatous reaction may result in variable imaging appearances on follow up MRI, including focal areas of enhancement and restricted diffusion, both of which may simulate residual disease following neuro-oncological interventions. Unsurprisingly, many terms have been introduced to describe foreign body-related inflammatory pseudo-tumours, and granulomatous reaction triggered by haemostatic agents. Awareness of this phenomenon is crucial; in fact after neuro-oncology procedures the determination of the presence of residual disease greatly impacts management plan and prognostication (45). For this reason careful evaluation of diffusion-weighted sequences is indicated in all postoperative imaging protocols, especially if the primary tumour shows no evidence of restricted diffusion (46).

\section{Intentionally retained foreign bodies}

Depending on the type of surgical intervention many different types of foreign bodies might be intentionally left in the surgical site, those include reservoirs, intraparenchymal/subdural/extradural probes for intracranial pressure monitoring (Figure 8), microdialysis catheters, deep sited electrodes or cortical stereoelectroencephalography (stereoEEG) grids, surgical drainages, etc. On one hand, this highlights the importance of good documentation in the operative notes, on the other hand it also demonstrates how an effective communication 
between the requesting clinician and the reporting radiologist can facilitate reporting and help addressing clinical questions about accuracy of the surgical trajectory/ placement. Such latter aspect can oftentimes be answered by reviewing the preoperative planning (based on CT, MRI and angiography image fusion), and intraoperative imaging (for instance that acquired through fluoroscopy and ultrasound) which are usually stored in the hospital imaging database $(47,48)$. Furthermore, it should be noted that any intentionally retained foreign-body could potentially cause inflammatory granuloma, resulting from the variable amount of acute and chronic inflammatory cells, collagen deposition, and reactive vascular proliferation, surrounding it. Therefore, the reporting radiologist should be mindful of the potential pitfalls related to such biologic reaction to foreign bodies and orientate clinicians regarding need for further monitoring or removal.

\section{Machine learning in neuroradiology reporting}

During the last decade, several progresses in imaging digitalisation and analysis, led to a growing interest in the use of artificial intelligence as a way to standardise image diagnosis and prognosis within the area of neuroradiology $(49,50)$. More than 1,000 papers have been published over the last five years (2015-2020) on subject classification and prediction focused on multiple brain disorders. However, to date, the majority of research trend and current status have not been clearly illuminated in the field of paediatric neuroradiology (50).

The field of radiomics, which describes a broad set of computational methods for extracting quantitative features from radiographic images will certainly be a game changer even in the management of paediatric neurosurgery, not only with regards to the initial diagnosis but also for postoperative assessment and follow up purposes. For now, the major challenges remain the need for methodological developments to further optimise feature extraction and provide rapid information flow in clinical settings. Equally important, will be to detect clinically useful, predictive radiomics properties and clearly link them to meaningful biologic characteristics and qualitative imaging properties familiar to neuroradiologists (51). Only 0.01 of the $>400$ clinical trials on the safety and efficacy of diagnostic and prognostic value of machine learning in various fields of radiology are specific for paediatric neuroradiology: NCT04217044; NCT04215211; NCT04215211;
NCT04153045. Interestingly, almost all of them revolve around the preoperative and postoperative management of patients harbouring intracranial tumours. Despite the above, it is sensible to forecast that automated image extraction, analysis and prediction will soon support clinical decision-making in any aspect of the paediatric arena too.

\section{Limitations of this study}

Two types of limitations, both considered and accepted at time of designing it, affect this study: the first regards the management of craniosynostosis, one area of paediatric neurosurgery not covered in this review; the second regards issues revolving around medico-legal liability of paediatric neuroradiologists.

First, craniosynostosis affects 1 in 2,100-2,500 live births, and its most common presentation are cranial malformations in the first year of life (52). The condition often involves intra-cranial pressure and impaired brain growth (53). Three-dimensional CT is the de facto imaging standard to diagnose craniosynostosis based on the observation of the fused sutures; nonetheless MRI brain is routinely conducted preoperatively to rule out syndromic conditions and plan surgical correction (54). The reason why craniosynostosis was not included in this review is that, due to concerns about exposing children to unnecessary sedation and potentially harmful radiation, the relevance of post-surgical imaging is considered negligible by the neurosurgical community, unless suboptimal treatment, postoperative complications or late relapse are suspected.

Second, while it is true that neuroradiologists are liable for: (I) their decisions and actions, (II) their competence and continuous medical education, and (III) their own equipment and staff, such liability might not only be questioned in cases of litigation, but also in internal investigations following serious events (1,55). One limitation of this review is that aspects related to clinical competence, adherence to international guidelines and informed patient consent were purposely not included. The goal was instead educational, in that we tried to provide a practical guideline for postoperative reporting to paediatric neuroradiology trainees and fellows. Further reading is therefore suggested to all readers to ensure that all behaviours and decisionmaking processes are in keeping with the medico-legal standards of the countries of their individual radiological practice. 


\section{Conclusions}

The exemplificative cases and the review of the literature provided in this article offer a summary of the normal and abnormal radiological patterns commonly found after three types of paediatric neurosurgical procedures: ventricular access, supratentorial craniotomies, and posterior fossa decompression. Whereas the iconography enriching this article can serve as a reference for the day-to-day paediatric neuroimaging activity, the articles included in this review will help trainees and fellows to deepen these topics and expand their knowledge in preparation for written boards.

Various forces will continue to influence this radiological subspecialty; to this regards, the impact of relentless technical advancements of neuroimaging equipment, including hardware and software, seems to be particularly strong. What will not change though is the need for continuous and effective cross talk among clinicians and diagnostic paediatric neuroradiologists to ensure safe and appropriate clinical management of paediatric patients.

\section{Acknowledgments}

We are grateful to our graphic artist Dr Lara Prisco (@ PriscoLara) for her drawings which are complementing and enriching the postoperative images presented in this article. Funding: None.

\section{Footnote}

Provenance and Peer Review: This article was commissioned by the Guest Editor (Felice D'Arco) for the series "Pediatric Neuroradiology for Trainees and Fellows: An Updated Practical Guide" published in Translational Pediatrics. The article has undergone external peer review.

Conflicts of Interest: All authors have completed the ICMJE uniform disclosure form (available at http:// dx.doi.org/10.21037/tp-20-484). The series "Pediatric Neuroradiology for Trainees and Fellows: An Updated Practical Guide" was commissioned by the editorial office without any funding or sponsorship. The authors have no other conflicts of interest to declare.

Ethical Statement: The authors are accountable for all aspects of the work in ensuring that questions related to the accuracy or integrity of any part of the work are appropriately investigated and resolved
Open Access Statement: This is an Open Access article distributed in accordance with the Creative Commons Attribution-NonCommercial-NoDerivs 4.0 International License (CC BY-NC-ND 4.0), which permits the noncommercial replication and distribution of the article with the strict proviso that no changes or edits are made and the original work is properly cited (including links to both the formal publication through the relevant DOI and the license). See: https://creativecommons.org/licenses/by-nc-nd/4.0/.

\section{References}

1. Picard L. Medicolegal aspects in neuroradiologic emergencies. J Neuroradiol 2004;31:340-6.

2. Baethge C, Goldbeck-Wood S, Mertens S. SANRA-a scale for the quality assessment of narrative review articles. Res Integr Peer Rev 2019;4:5.

3. Láng J, Ganau M, Prisco L, et al. Syndrome of trephined-underestimated and poorly understood complication after decompressive craniectomy. A trephined-szindróma - egy alulbecsült és kevéssé értett komplikáció dekompresszív craniectomia után. Ideggyogy Sz 2016;69:227-32.

4. Flannery AM, Duhaime AC, Tamber MS, et al. Pediatric hydrocephalus: systematic literature review and evidencebased guidelines. Part 3: Endoscopic computer-assisted electromagnetic navigation and ultrasonography as technical adjuvants for shunt placement. J Neurosurg Pediatr 2014;14 Suppl 1:24-9.

5. Morone PJ, Dewan MC, Zuckerman SL, et al. Craniometrics and Ventricular Access: A Review of Kocher's, Kaufman's, Paine's, Menovksy's, Tubbs', Keen's, Frazier's, Dandy's, and Sanchez's Points. Oper Neurosurg (Hagerstown) 2020;18:461-9.

6. Coulter IC, Kulkarni AV, Sgouros S, et al. Cranial and ventricular size following shunting or endoscopic third ventriculostomy (ETV) in infants with aqueductal stenosis: further insights from the International Infant Hydrocephalus Study (IIHS). Childs Nerv Syst 2020;36:1407-14.

7. Tan RMR, Ganau M, Jeelani NUO, et al. Central nervous system aspergillosis resembling haemorrhagic brain infarct in a paediatric leukaemia patient. Br J Haematol 2017;178:642-5.

8. Ganau M, Talenti G, D'Arco F. Teaching NeuroImages: Radiologic features of septo-optic dysplasia plus syndrome. Neurology 2018;91:e2200-1.

9. Zairi F, Le Rhun E, Bertrand N, et al. Complications 
related to the use of an intraventricular access device for the treatment of leptomeningeal metastases from solid tumor: a single centre experience in 112 patients. J Neurooncol 2015;124:317-23.

10. Parikh D, Foroughi M, Nannapaneni R, et al. Is the routine placement of a CSF reservoir following endoscopic third ventriculostomy justified? Br J Neurosurg 2009;23:521-3.

11. Aquilina K, Edwards RJ, Pople IK. Routine placement of a ventricular reservoir at endoscopic third ventriculostomy. Neurosurgery 2003;53:91-6; discussion 96-7.

12. Aljohani H, Romano A, Iaccarino C, et al. Pure Endoscopic Management of Fourth Ventricle Arachnoid Cyst: Case Report and Literature Review. Asian J Neurosurg 2018;13:184-7.

13. Chibbaro S, Cebula H, Scholly J, et al. Pure endoscopic management of epileptogenic hypothalamic hamartomas. Neurosurg Rev 2017;40:647-53.

14. Boukobza M, Cebula H, Pop R, et al. Cystic meningioma: radiological, histological, and surgical particularities in 43 patients. Acta Neurochir (Wien) 2016;158:1955-64.

15. Chibbaro S, Ganau M, Gubian A, et al. The Role of Endoscopic Endonasal Approach in the Multimodal Management of Giant Pituitary Adenoma: Case Report and Literature Review. Asian J Neurosurg 2018;13:888-92.

16. Ganau M, Syrmos NC, D'Arco F, et al. Enhancing contrast agents and radiotracers performance through hyaluronic acid-coating in neuroradiology and nuclear medicine. Hell J Nucl Med 2017;20:166-8.

17. Graziano F, Ganau M, Meccio F, et al. The Transcallosal Anterior Interfoniceal Approach: A Microsurgical Anatomy Study. J Neurol Surg B Skull Base 2015;76:183-8.

18. Soleman J, Ber R, Constantini S, et al. The interhemispheric approach in children: our experience and review of the literature. Childs Nerv Syst 2019;35:445-52.

19. Aryan HE, Ozgur BM, Jandial R, et al. Complications of interhemispheric transcallosal approach in children: review of 15 years experience. Clin Neurol Neurosurg 2006;108:790-3.

20. Zhang YQ, Ma ZY, Wu ZB, et al. Radical resection of 202 pediatric craniopharyngiomas with special reference to the surgical approaches and hypothalamic protection. Pediatr Neurosurg 2008;44:435-43.

21. D'Arco F, Khan F, Mankad K, et al. Differential diagnosis of posterior fossa tumours in children: new insights. Pediatr Radiol 2018;48:1955-63.

22. Cochrane DD, Gustavsson B, Poskitt KP, et al. The surgical and natural morbidity of aggressive resection for posterior fossa tumors in childhood. Pediatr Neurosurg 1994;20:19-29.

23. Catsman-Berrevoets C, Patay Z. Cerebellar mutism syndrome. Handb Clin Neurol 2018;155:273-288.

24. Liu JF, Dineen RA, Avula S, et al. Development of a pre-operative scoring system for predicting risk of postoperative paediatric cerebellar mutism syndrome. Br J Neurosurg 2018;32:18-27.

25. Meoded A, Jacobson L, Liu A, et al. Diffusion Tensor Imaging Connectomics Reveals Preoperative Neural Connectivity Changes in Children with Postsurgical Posterior Fossa Syndrome. J Neuroimaging 2020;30:192-7.

26. D'Arco F, Hanagandi P, Ganau M, et al. Neuroimaging Findings in Lysosomal Disorders: 2018 Update. Top Magn Reson Imaging 2018;27:259-74.

27. Ganau M, Spinelli R, Tacconi L. Complex developmental abnormality of the atlas mimicking a Jefferson fracture: Diagnostic tips and tricks. J Emerg Trauma Shock 2013;6:47-9.

28. Singh S, Sardhara J, Raiyani V, et al. Lessons learned from surgical management of craniovertebral instability in Morquio syndrome: A series of four unusual cases. J Craniovertebr Junction Spine 2020;11:243-7.

29. Williams N, Narducci A, Eastwood DM, et al. An Evidence-based Approach to the Management of Children With Morquio A Syndrome Presenting With Craniocervical Pathology. Spine (Phila Pa 1976) 2018;43:E1443-53.

30. Menezes AH. Craniovertebral junction neoplasms in the pediatric population. Childs Nerv Syst 2008;24:1173-86.

31. Massimi L, Thompson DNP. Introduction to the special edition from the Editors Pediatric Chiari I malformationsearching for clarity amidst myth, misnomer, and misunderstanding. Childs Nerv Syst 2019;35:1651-2.

32. D'Arco F, Ganau M. Which neuroimaging techniques are really needed in Chiari I? A short guide for radiologists and clinicians. Childs Nerv Syst 2019;35:1801-8.

33. Pereira EA, Magdum SA. Foramen magnum decompression - from hygromas to hydrocephalus. Br J Neurosurg 2016;30:355.

34. Pereira EA, Steele LF, Magdum SA. Recurrent subdural hygromas after foramen magnum decompression for Chiari Type I malformation. Br J Neurosurg 2014;28:396-9.

35. Tam SKP, Brodbelt A, Bolognese PA, et al. Posterior fossa decompression with duraplasty in Chiari malformation type 1: a systematic review and meta-analysis. Acta Neurochir (Wien) 2021;163:229-38.

36. Chotai S, Chan EW, Ladner TR, et al. Timing of 
syrinx reduction and stabilization after posterior fossa decompression for pediatric Chiari malformation type I. J Neurosurg Pediatr 2020. [Epub ahead of print]. doi: 10.3171/2020.2.PEDS19366.

37. Pearce K, Dixon L, D'Arco F, et al. Epilepsy surgery in children: what the radiologist needs to know. Neuroradiology 2020;62:1061-78.

38. Simonato D, Ganau M, Feltracco P, et al. Mechanical thrombectomy in a pediatric patient with sedation aided by contralateral intra-arterial propofol injection: feasibility in an extreme condition. Childs Nerv Syst 2020. [Epub ahead of print]. doi: 10.1007/s00381-020-04872-4

39. Ghoneim AA, Azer MS, Ghobrial HZ, et al. Awakening properties of isoflurane, sevoflurane, and desflurane in pediatric patients after craniotomy for supratentorial tumours. J Neurosurg Anesthesiol 2015;27:1-6.

40. Kemaloglu S, Ozkan U, Bukte Y, et al. Timing of shunt surgery in childhood tuberculous meningitis with hydrocephalus. Pediatr Neurosurg 2002;37:194-8.

41. Chen Y, Meng X, Ma L, et al. Contemporary management of brain arteriovenous malformations in mainland China: a web-based nationwide questionnaire survey. Chin Neurosurg J 2020;6:26.

42. Ganau M, Graziano F, Iacopino D. Advanced Hemostatics in the Management of Cerebral Dural Sinus Lacerations. Neurosurgery 2015;77:E670-73.

43. Ganau M, Nicassio N, Tacconi L. Postoperative aseptic intracranial granuloma: the possible influence of fluid hemostatics. Case Rep Surg 2012;2012:614321.

44. Graziano F, Certo F, Basile L, et al. Autologous fibrin sealant (Vivostat@) in the neurosurgical practice: Part I: Intracranial surgical procedure. Surg Neurol Int 2015;6:77.

45. Steinbok P, Hentschel S, Cochrane DD, et al. Value of postoperative surveillance imaging in the management of children with some common brain tumors. J Neurosurg 1996;84:726-32.

46. Staglianò S, D'Arco F, Tan AP, et al. Haemostatic material
(Surgicel®) mimicking residual tumour: magnetic resonance imaging findings in operated pediatric neurooncology cases. Quant Imaging Med Surg 2018;8:971-8.

47. Ganau M, Ligarotti GK, Apostolopoulos V. Real-time intraoperative ultrasound in brain surgery: neuronavigation and use of contrast-enhanced image fusion. Quant Imaging Med Surg 2019;9:350-8.

48. Ganau M, Syrmos N, Martin AR, et al. Intraoperative ultrasound in spine surgery: history, current applications, future developments. Quant Imaging Med Surg 2018;8:261-7.

49. Sboarina A, Foroni RI, Minicozzi A, et al. Software for hepatic vessel classification: feasibility study for virtual surgery. Int J Comput Assist Radiol Surg 2010;5:39-48.

50. Sakai K, Yamada K. Machine learning studies on major brain diseases: 5-year trends of 2014-2018. Jpn J Radiol 2019;37:34-72.

51. Zhou M, Scott J, Chaudhury B, et al. Radiomics in Brain Tumor: Image Assessment, Quantitative Feature Descriptors, and Machine-Learning Approaches. AJNR Am J Neuroradiol 2018;39:208-16.

52. Thomas GP, Johnson D, Byren JC, et al. The incidence of raised intracranial pressure in nonsyndromic sagittal craniosynostosis following primary surgery. J Neurosurg Pediatr 2015;15:350-60.

53. Wall SA, Thomas GP, Johnson D, et al. The preoperative incidence of raised intracranial pressure in nonsyndromic sagittal craniosynostosis is underestimated in the literature. J Neurosurg Pediatr 2014;14:674-81.

54. Ganau M, Paris M, Uff C. Images in Neuroscience: Calvarial hyperostosis associated with multiple intracranial tumours. J Clin Neurosci. [Epub ahead of print]. doi: 10.1016/j.jocn.2018.01.029.

55. Shen FX, Wolf SM, Gonzalez RG, et al. Ethical Issues Posed by Field Research Using Highly Portable and Cloud-Enabled Neuroimaging. Neuron 2020;105:771-5.
Cite this article as: Ganau M, Magdum SA, Calisto A. Preoperative imaging and post-operative appearance of standard paediatric neurosurgical approaches: a training guide for neuroradiologists. Transl Pediatr 2021;10(4):1231-1243. doi: $10.21037 /$ tp-20-484 